\title{
Thermoelectric Figure-of-Merit of Fully Dense Single-Crystalline SnSe
}

Pai-Chun Wei, ${ }^{\dagger \dagger \dagger}$ Sriparna Bhattacharya, ${ }^{\ddagger}$ Yu-Fei Liu, ${ }^{\ddagger}$ Fengjiao Liu, ${ }^{\ddagger}$ Jian He, ${ }^{\ddagger}$ Yung-Hsiang Tung, Chun-Chuen Yang, ${ }^{\S}$ Cheng-Rong Hsing," Duc-Long Nguyen, $\|, \Phi, \nabla$ Ching-Ming Wei, ${ }^{\| P}$ Mei-Yin Chou, Yen-Chung Lai, ${ }^{\perp}$ Tsu-Lien Hung, ${ }^{\dagger}$ Syu-You Guan, ${ }^{\dagger, \#}$ Chia-Seng Chang, ${ }^{\dagger, \#}$ Hsin-Jay Wu, ${ }^{\circ}$ Chi-Hung Lee, ${ }^{\text {II }}$ Wen-Hsien Li, ${ }^{\text {, }}$ Raphael P. Hermann, ${ }^{\circledR}$ Yang-Yuan Chen, ${ }^{*}$, and Apparao M. Rao*, $* 0$

${ }^{\dagger}$ Institute of Physics, Academia Sinica, Taipei 11529, Taiwan, Republic of China

${ }^{\ddagger}$ Department of Physics and Astronomy, Clemson Nanomaterials Institute, Clemson University, Clemson 29634-0978, United States

${ }^{\S}$ Department of Physics, Chung Yuan Christian University, Chung-Li 32023, Taiwan, Republic of China

"Institute of Atomic and Molecular Sciences, Academia Sinica, Taipei 10617, Taiwan, Republic of China

${ }^{\perp}$ National Synchrotron Radiation Research Center, Hsin-Chu 30076, Taiwan, Republic of China

\#Department of Physics, National Taiwan University, Taipei 10617, Taiwan, Republic of China

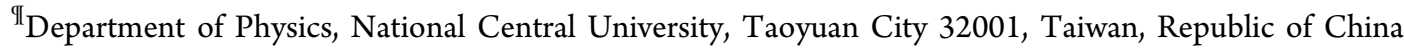

${ }^{\nabla}$ Molecular Science and Technology Program, Taiwan International Graduate Program, Academia Sinica, Taipei 11529, Taiwan, Republic of China

${ }^{O}$ Department of Materials Science and Engineering, National Chiao Tung University, Hsinchu 30010, Taiwan, Republic of China

Materials Science and Technology Division, Oak Ridge National Laboratory, Oak Ridge, Tennessee 37831, United States

${ }^{\dagger \dagger}$ Computer, Electrical, and Mathematical Sciences and Engineering Division, King Abdullah University of Science and Technology (KAUST), Thuwal 23955-6900, Saudi Arabia

\section{Supporting Information}

ABSTRACT: Single-crystalline SnSe has attracted much attention because of its record high figure-of-merit $Z T \approx 2.6$; however, this high $Z T$ has been associated with the low mass density of samples which leaves the intrinsic $Z T$ of fully dense pristine $\mathrm{SnSe}$ in question. To this end, we prepared high-quality fully dense SnSe single crystals and performed detailed structural, electrical, and thermal transport measurements over a wide temperature range along the major crystallographic directions. Our single crystals were fully dense and of high purity as confirmed via high statistics ${ }^{119} \mathrm{Sn}$ Mössbauer spectroscopy that revealed $<0.35$ at. \% $\mathrm{Sn}(\mathrm{IV})$ in pristine $\mathrm{SnSe}$. The temperature-dependent heat capacity $\left(C_{\mathrm{p}}\right)$ provided evidence for the displacive second-order phase transition from Pnma to $\mathrm{Cmcm}$ phase at $T_{\mathrm{c}} \approx$ $800 \mathrm{~K}$ and a small but finite Sommerfeld coefficient $\gamma_{0}$ which implied the presence of a finite Fermi surface. Interestingly, despite its strongly temperature-dependent band gap inferred from density functional theory calculations, SnSe behaves like a

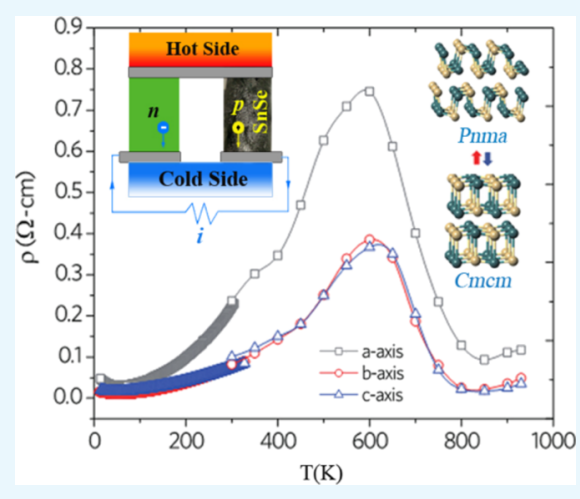
low-carrier-concentration multiband metal below $600 \mathrm{~K}$, above which it exhibits a semiconducting behavior. Notably, our high-quality single-crystalline SnSe exhibits a thermoelectric figure-of-merit ZT $\sim 1.0$, $\sim 0.8$, and $\sim 0.25$ at $850 \mathrm{~K}$ along the $b, c$, and $a$ directions, respectively.

\section{INTRODUCTION}

Thermoelectric (TE) materials enable a green conversion of waste heat to electrical energy. This conversion efficiency is mainly determined by the dimensionless figure-of-merit $Z T=$ $S^{2} T / \rho \kappa$, where $\rho, S, T$, and, $\kappa$ are the electrical resistivity, Seebeck coefficient, absolute temperature, and thermal conductivity, respectively. In the simplest case, $\kappa=\kappa_{\mathrm{E}}+\kappa_{\mathrm{L}}$ where $\kappa_{\mathrm{E}}$ and $\kappa_{\mathrm{L}}$ are the electronic and lattice contributions, respectively, and the power factor, $\mathrm{PF}=S^{2} / \rho$, is often defined to measure the electrical performance of the material. Several effective approaches for enhancing ZT have been devised, for example, reduced dimensionality, ${ }^{1}$ high crystal structure

Received: November 28, 2018

Accepted: February 14, 2019

Published: March 19, 2019 
complexity, ${ }^{2}$ band convergence, ${ }^{3}$ defect engineering, ${ }^{4}$ anharmonicity, ${ }^{5}$ and cagey semiconductors. ${ }^{6}$ To date, state-of-the-art TE materials are predominantly semimetals or semiconductors with high crystal symmetry, small electronegativity difference among constituent elements, and complex unit cell with the presence of heavy elements (e.g., $\mathrm{Bi}, \mathrm{Pb}$, etc.). In this regard, pristine $\mathrm{SnSe}$ is an outlier among these materials and has been the subject of much research because of a high $Z T \approx 2.6$ that was reported in early studies by Zhao et al., ${ }^{7}$ and a $Z T \approx 1$ in subsequent studies.

Unlike other high-performance TE materials such as $\mathrm{Bi}_{2} \mathrm{Te}_{3}$, $\mathrm{CoSb}_{3}, \mathrm{PbTe}$, and $\mathrm{SiGe}$ that have hexagonal or cubic crystal structures, $\mathrm{SnSe}$ has a relatively lower symmetry orthorhombic structure which undergoes a second-order Pnma (\#62)-to$\mathrm{Cmcm}$ (\#63) displacive phase transition at $T_{\mathrm{c}} \approx 800 \mathrm{~K}$. ${ }^{9}$ In addition, although SnSe has a low number of atoms per unit cell and contains no heavy elements such as $\mathrm{Bi}$ and $\mathrm{Pb}$, it surprisingly exhibits an exceptionally low $\kappa_{\mathrm{L}}$ in its pristine form. It is this ultralow $\kappa_{\mathrm{L}}$ that underscores the reported record-high $Z T .^{7}$ On the one hand, strong anharmonicity has been confirmed in $\mathrm{SnSe}$, and recent inelastic neutron scattering measurements combined with first-principle simulations have provided further insights into the origin of the anharmonicity in $\mathrm{SnSe}$, viz., a coupling of the lattice distortion below $T_{\mathrm{c}}$ to the instability of the electronic structure. ${ }^{10,11}$ On the other hand, it is an open question as to whether strong anharmonicity alone can account for the observed low $\kappa_{\mathrm{L}}$ in ref 7 because it has been argued in the literature that the SnSe samples therein were of low packing density $(\sim 88 \%$ of the theoretical density). ${ }^{8}$ Moreover, contrary to our present understanding of phonon scattering mechanisms in solids, $\kappa_{\mathrm{L}}$ of polycrystalline $\mathrm{SnSe}^{12,13}$ was higher than that reported by Zhao et al. ${ }^{7}$ Furthermore, in their study of fully dense SnSe single crystals, Ibrahim et al. ${ }^{14}$ reported an electrical transport behavior that is distinct from the earlier findings of Zhao et al. ${ }^{7}$ and Wang et al., ${ }^{15}$ which is possibly due to different growth conditions leading to different hole concentrations. ${ }^{16}$ In a recent study, Wang et al. ${ }^{17}$ also reported that the hole-doping mechanism in $\mathrm{SnSe}$ and resulting electron conduction behavior were sensitive to synthesis parameters that led to two unique defects, namely, the formation of $\mathrm{SnSe}_{2}$ and/or dislocations, albeit no strong evidence for these defects was provided in their study. Last, although $\mathrm{SnSe}$ is an intrinsic p-type semiconductor with a band gap $\sim 0.9-1.0 \mathrm{eV}$ and a low carrier concentration $\sim 10^{17}$ $\mathrm{cm}^{-318-20}$ in the Pnma phase, the high $Z T$ in pristine SnSe is achieved through its high carrier concentration in the $\mathrm{Cmcm}$ phase $\left.\left(\sim 10^{19} \mathrm{~cm}^{-3}\right)\right)^{7}$ The p-type nature of SnSe has been attributed to native $S n$ vacancies. ${ }^{21}$

Theoretical studies of pristine $\mathrm{SnSe}$ have shown the temperature evolution of the Pnma crystal phase that leads to significant changes in the electronic band structure. ${ }^{18,19}$ Thus, it is imperative to experimentally investigate the interplay between a small yet robust Fermi surface as inferred from a finite Sommerfeld coefficient and the temperaturedependent band gap by means of electrical and thermal measurements over a wide temperature range. Here, we report detailed temperature-dependent structural, electronic, and thermal properties along the three major crystallographic orientations of fully dense single-crystalline SnSe. A unique aspect of this study is that in our density functional theory (DFT) calculations, we used the experimentally determined temperature-dependent structural parameters to gain a deeper understanding of the fundamental mechanisms at play in $\mathrm{SnSe}$ and to describe their behavior over a wide range of temperatures $(10-900 \mathrm{~K})$. We conclude that fully dense pristine single-crystalline $\mathrm{SnSe}$, with $<0.35$ at. \% $\mathrm{Sn}(\mathrm{IV})$ as revealed by high precision ${ }^{119} \mathrm{Sn}$ Mössbauer spectroscopy, exhibits a $Z T \approx 0.8-1$ above $\sim 800 \mathrm{~K}$.

\section{RESULTS AND DISCUSSION}

2.1. Structural Properties. Two representative SnSe single crystals grown by the Bridgman method are shown in Figure 1A. The crystals could be cleaved easily along the $a$
A

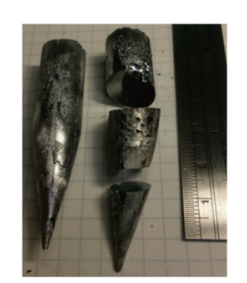

C

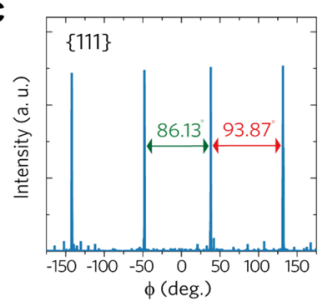

$\mathrm{E}$

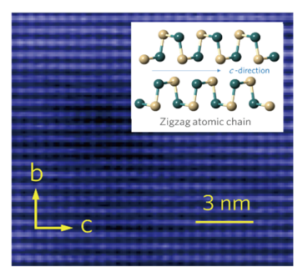

B

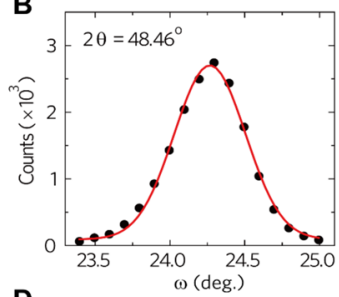

D

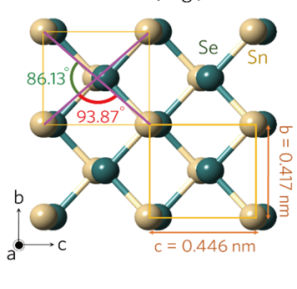

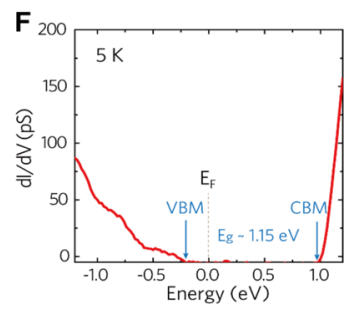

Figure 1. Structural characterization of fully dense single-crystalline SnSe. (A) Bridgman-grown SnSe crystals with the crystal direction along the (100) face. (B) Neutron rocking curve of single-crystalline SnSe $(19 \mathrm{~g})$ measured at room temperature. The fwhm is estimated to be $0.48(1)^{\circ}$, indicating the high crystallinity of our Bridgman-grown SnSe samples. (C) X-ray phi-scan profile of SnSe (111) reflection. (D) Crystal structure of Pnma-SnSe along the a direction. (E) STM topography of SnSe (100) surface obtained at a bias voltage of $1.6 \mathrm{~V}$ and $200 \mathrm{pA}$ constant current at $5 \mathrm{~K}$, inset: zigzag chains of $\mathrm{Sn}-\mathrm{Se}$ atoms. (F) Tunneling spectrum $\mathrm{d} I / \mathrm{d} V$ obtained at $4.8 \mathrm{~K}$ on (100) surface, where VBM and CBM are valence band maximum and the conduction band minimum, respectively. $E_{\mathrm{F}}$ and $E_{\mathrm{g}}$ are the Fermi energy and the energy band gap, respectively.

direction (because of the weak van der Waals bonds), yielding atomically flat surfaces that exhibit a metallic luster. ${ }^{22}$ The room-temperature density of $\mathrm{SnSe}$ crystals as determined from the Archimedes method $\left(6.16 \pm 0.01 \mathrm{~g} \mathrm{~cm}^{-3}\right)$ and gas pycnometry $\left(6.15 \pm 0.06 \mathrm{~g} \mathrm{~cm}^{-3}\right)$ on both as-grown ingot and cut pieces was consistent with the density estimated from our synchrotron X-ray diffraction (XRD) measurements ( $\sim 6.14 \mathrm{~g}$ $\mathrm{cm}^{-3}$ ) which is $\sim 100 \%$ of the theoretical density. Figure $1 \mathrm{~B}$ shows the neutron rocking curve of a $19 \mathrm{~g} \mathrm{SnSe}$ crystal at $2 \theta=$ $48.46^{\circ}$. The full width at half-maximum (fwhm) is estimated to be $0.48(1)^{\circ}$, revealing the excellent quality of monocrystalline $\mathrm{SnSe}$ from the Bridgman method. The XRD pattern of our SnSe crystal revealed four distinct (111) peaks and is shown in Figure 1C. From the XRD phi-scan, the in-plane orientation of SnSe single crystals can be explicitly identified, for example, the 
angles between two adjacent (111) peaks were found to be $86.13^{\circ}$ and $93.87^{\circ}$ (Figure 1C,D), in good agreement with previous neutron scattering results. ${ }^{9}$ It is well known that the $\mathrm{Sn}-\mathrm{Se}$ atomic layers are corrugated (because of the zigzagged $\mathrm{Sn}-\mathrm{Se}$ atomic chains along the $c$ direction as shown in the inset figure of Figure 1E), and hence, the topography of the scanning tunneling spectrometer (STM) image of the (100) surface in Figure $1 \mathrm{E}$ collected at $5 \mathrm{~K}$ reveals only the top $\mathrm{Sn}$ layer of the crystal, which is defect-free. Figure $1 \mathrm{~F}$ shows a representative differential-conductance, $\mathrm{d} I / \mathrm{d} V$ spectrum for $\mathrm{SnSe}$, which represents the density of state (DOS) as a function of energy. Clearly, the valence band maximum (VBM) is close to the Fermi level $\left(E_{\mathrm{F}}=0\right)$, leading to the p-type nature of $\mathrm{SnSe}$, and the band gap is estimated to be $\sim 1.15 \mathrm{eV}$ at $\sim 5 \mathrm{~K}$.

2.2. Mössbauer Spectroscopy. A detailed ${ }^{119} \mathrm{Sn}$ Mössbauer spectral study was performed on a single-crystalline $\mathrm{SnSe}$ sample (Figure 2) to confirm their stoichiometry and high

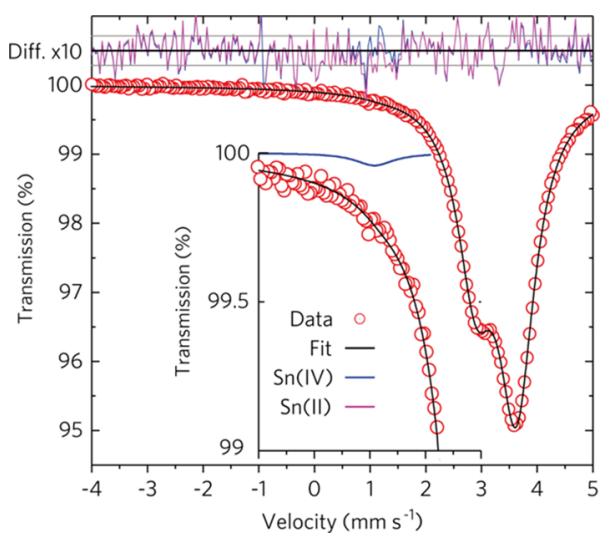

Figure 2. Room-temperature Mössbauer spectra of fully dense SnSe. Top: difference plot for fits with a single SnSe doublet fit (purple) and a fit with an additional $\mathrm{Sn}(\mathrm{IV}$ ) singlet (blue). The scale is magnified by a factor 10 . The gray lines represent the $\pm 1 \sigma$ deviation calculated from the counting statistics. Center: Mössbauer spectral data, red dots, and total fit, black line, for the model with SnSe and a minor $\mathrm{Sn}$ (IV) component. Inset: vertically enlarged spectrum and fit components in the -1 to $2 \mathrm{~mm} / \mathrm{s}$ range. The $\mathrm{Sn}(\mathrm{IV})$ minority component displayed in blue amounts to 0.35 at. \%. The SnSe doublet is displayed in magenta. Data points for data are scaled to match the error bar.

crystalline quality. No measurable amount of oxidation or $\mathrm{SnO}_{2}$, which would appear as a signal at $0 \mathrm{~mm} / \mathrm{s}$, was observed. A very small minority component which is other than $\mathrm{SnSe}$ $[\sim 0.35(5)$ at. $\% \mathrm{Sn}(\mathrm{IV})$ in $\mathrm{SnSe}]$ is observed in the spectrum and was modeled with the spectral parameters of $\mathrm{SnSe}_{2}$ (an isomer shift of $1.3 \mathrm{~mm} / \mathrm{s}$, a quadrupole splitting of $0 \mathrm{~mm} / \mathrm{s}$, and a linewidth of $0.9 \mathrm{~mm} / \mathrm{s}) .{ }^{23}$ The impurity phase could also be attributed to defective local coordination that yields an isomer shift similar to $\mathrm{Sn}(\mathrm{IV})$ in chalcogenide coordination. The main phase Mössbauer spectrum is an asymmetric doublet, which is also observed in powders ${ }^{24}$ but is more pronounced here because of the anisotropy of the singlecrystalline layers. An initial fit (with a simple doublet) yields the spectral parameters for the majority phase corresponding to $\mathrm{SnSe}{ }^{23,25}$ The isomer shift is $3.34(1) \mathrm{mm} / \mathrm{s}$, and the quadrupole splitting is $0.732(5) \mathrm{mm} / \mathrm{s}$, with a linewidth of $0.73(2) \mathrm{mm} / \mathrm{s}$. This sum of square residuals (SSR) for this fit is $296.0(0.1)$ for 256 data points and 7 fit parameters, $\left(\chi_{\text {red. }}^{2}=\right.$
1.19). The doublet asymmetry is given by a $36: 64$ ratio of intensity between the left and right lines, for SnSe. The difference plot with the initial fit, in magenta, top of Figure 2 indicates the presence of a small residual component around $0.9 \mathrm{~mm} / \mathrm{s}$. Hence, a broadened line corresponding to $\mathrm{SnSe}_{2}$ was included in the final fit. The difference plot with the final fit, in blue, top of Figure 2 indicates no further residual components, implying that the two-component fit suffices $[S S R=278.0(0.1), k=8$ parameters, $N=256$ data points, $\left.\chi_{\text {red. }}{ }^{2}=1.12\right]$. Figure 2 shows the data and the two-component fit for SnSe. The difference plot at the top is magnified by a factor 10 and the $\pm 1 \sigma$ deviation is depicted by gray lines. The inset to the figure enlarges the velocity range where the secondary doublet is observed. The majority SnSe phase component is displayed in magenta, the minority doublet is displayed in blue and the total fit is displayed in black. Note that to quantify the minority phase, we have taken into account the temperature dependence of the Lamb-Mössbauer factor for $\mathrm{Sn}$ in $\mathrm{SnSe}$ and $\mathrm{SnSe}_{2}$ as calculated from the partial density of $\mathrm{Sn}$ phonon states obtained experimentally ${ }^{11}$ and theoretically, ${ }^{26}$ respectively.

2.3. Temperature-Dependent Synchrotron XRD and Heat Capacity. Figure 3A shows the temperature-dependent evolution of synchrotron XRD patterns of SnSe powder. It is evident that several peaks split into two near the phase transition temperature ( $800 \mathrm{~K}$, from $\mathrm{Cmcm}$ to Pnma). The temperature-dependent lattice parameters obtained from XRD and neutron diffraction measurements are shown in Figure 3B. With increasing temperature, the two shorter lattice parameters (along $b$ and $c$ directions) in the Pnma phase approach each other and become nearly equal in the $\mathrm{Cmcm}$ phase, whereas the lattice parameter along the $a$ direction increases monotonically. All the three lattice parameters evolve continuously across $T_{\mathrm{c}}$ to a higher symmetry $\mathrm{Cmcm}$ phase. Interestingly, the lattice parameter along the $c$ direction decreases with the increasing temperature, indicative of a negative thermal expansion due to a strong coupling to the anharmonic phonon modes. ${ }^{11}$ To further elucidate this anharmonicity, the $C_{\mathrm{p}}$ of a fully dense SnSe crystal was measured from 0.45 to $875 \mathrm{~K}$ (Figure 3C). In addition to the continuity of unit cell volume at $800 \mathrm{~K}$ (inset in Figure 3C), the characteristic lambda-shaped $C_{\mathrm{p}}$ peak at $T_{\mathrm{c}} \approx 800 \mathrm{~K}$ is a signature of a second-order phase transition, ${ }^{27}$ the shape of which was retained through several temperature cycles. In the temperature range from $300 \mathrm{~K}$ to $T_{\mathcal{C}}$ the measured $C_{\mathrm{p}}$ is higher than the Dulong-Petit limit $\sim 49.9 \mathrm{~J}$ $\mathrm{mol}^{-1} \mathrm{~K}^{-1}$, signifying a strong anharmonicity at $T<T_{\mathcal{c}}$, prior to the onset of the lambda transition near $T_{\mathrm{c}}$. In contrast, the $C_{\mathrm{p}}$ attains the Dulong-Petit limit above $T_{\mathrm{c}}$. As shown in the inset in Figure 3C, the volume expansion largely retains the same positive temperature coefficient above $T_{\mathcal{c}}$, indicating the presence of anharmonicity above $T_{c}$ that is largely offset by the phonon softening in the $\mathrm{Cmcm}$ phase.

From a linear plot of $C_{\mathrm{p}} / T$ versus $T^{2}$ in the low temperature regime $(0.45-1 \mathrm{~K})$, a Debye temperature $\Theta_{\mathrm{D}} \approx 206 \mathrm{~K}$ was estimated using the relation $C_{\mathrm{p}}(T)=\gamma_{0} T+\beta T^{3}$ where a Sommerfeld coefficient $\gamma_{0} \approx(6.0 \pm 0.7) \times 10^{-5} \mathrm{~J} \mathrm{~mol}^{-1} \mathrm{~K}^{-2}$ and the lattice contribution to heat capacity $\beta \approx 4.4 \times 10^{-4} \mathrm{~J}$ $\mathrm{mol}^{-1} \mathrm{~K}^{-4}$ were obtained (Figure 3D). The $\gamma_{0}$ value of SnSe is comparable to that of $\mathrm{Bi}\left(\sim 4-8 \times 10^{-5} \mathrm{~J} \mathrm{~mol}^{-1} \mathrm{~K}^{-2}\right),{ }^{28}$ and an order of magnitude lower than that of copper $\left(\gamma_{\mathrm{Cu}}=6.95 \times\right.$ $10^{-4} \mathrm{~J} \mathrm{~mol}^{-1} \mathrm{~K}^{-2}$ ), implying a low DOS $\left(E_{\mathrm{F}}\right)$ and a small Fermi surface. This small but robust Fermi surface in SnSe could arise 

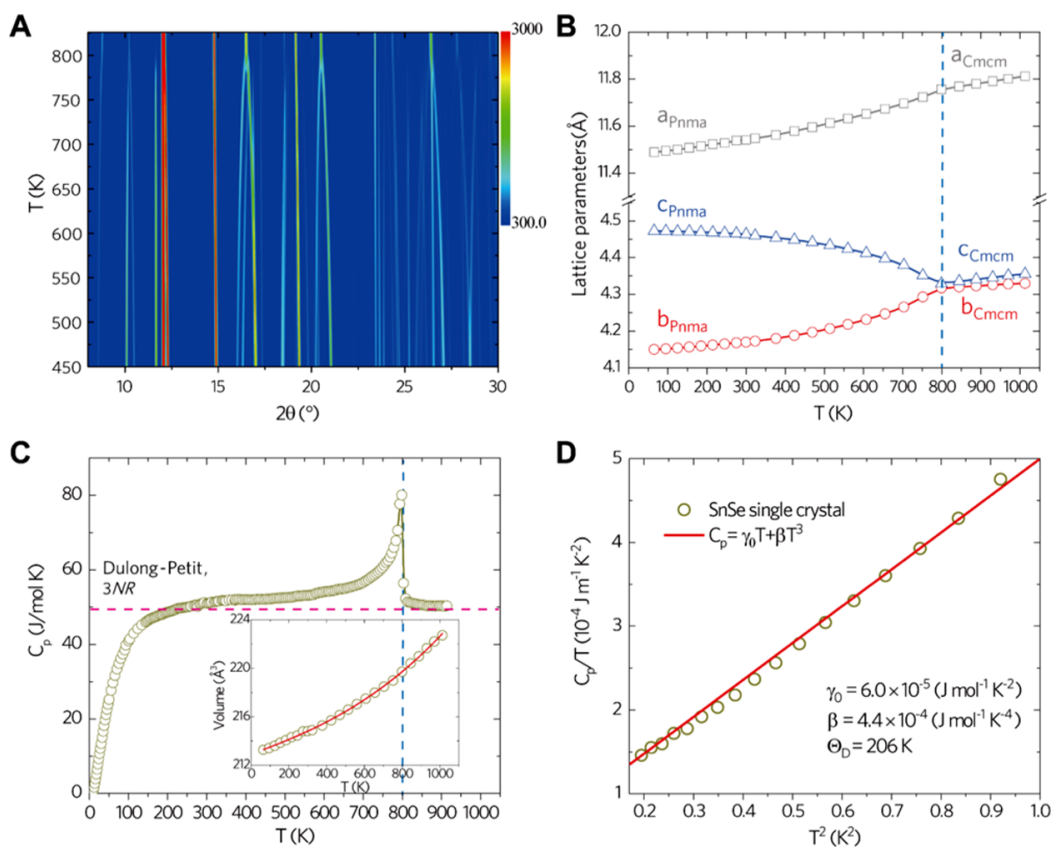

Figure 3. Temperature-dependent synchrotron XRD patterns and heat capacity data of SnSe. (A) Temperature-dependent XRD patterns showing the peak evolution with temperature. (B) Lattice parameters determined from Rietveld refinement and plotted as a function of temperature along $a$, $b$, and $c$ directions. (C) $C_{\mathrm{p}}$ as a function of temperature below and above $T_{\mathrm{c}}$ (vertical blue dashed line). Inset: unit cell volume plotted as a function of temperature. (D) Estimated Debye temperature $\left(\Theta_{\mathrm{D}}\right)$ from the plot of $C_{\mathrm{p}} / T$ vs $T^{2}$.

A

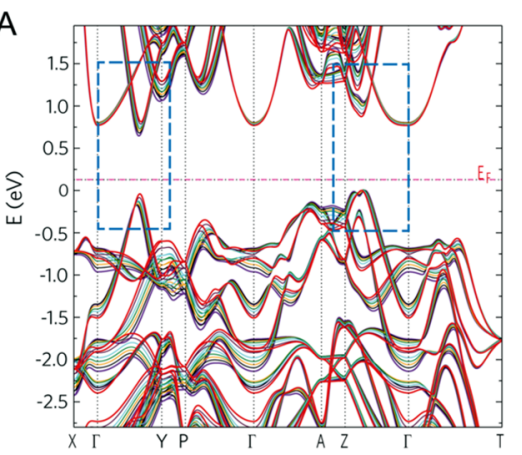

C

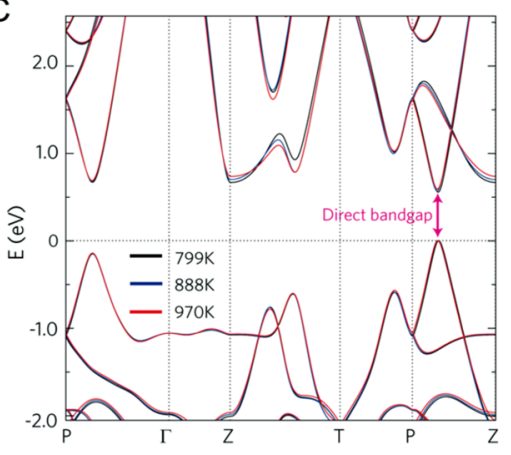

B

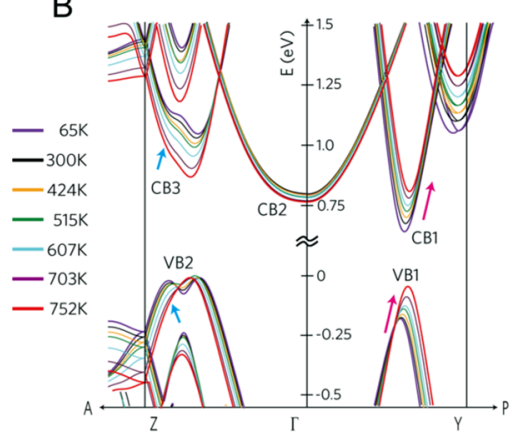

$\mathrm{D}$

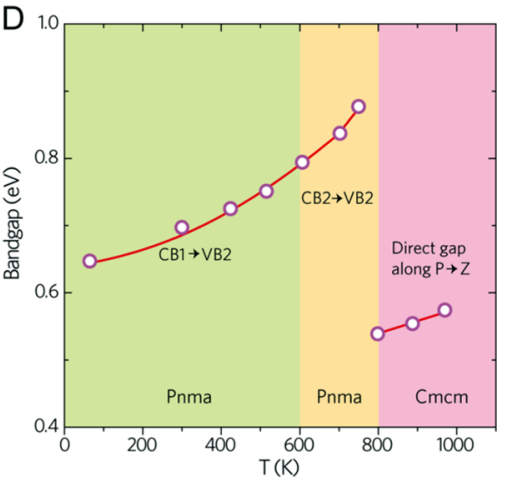

Figure 4. Band structure of SnSe using DFT. (A) Below and (C) above the phase transition, (B) expanded view of the VBM and CBM indicated in the dotted squares in (A). (D) Estimated energy band gap above and below the phase transition temperature.

from the presence of native defects, mainly Sn vacancies in this case, which are easy to form. ${ }^{29}$

2.4. Temperature-Dependent Band Structure and

Electronic Transport. Figure 4A,C shows the $\mathrm{SnSe}$ band structure $\left(\mathrm{DFT}+\mathrm{GW}_{0}\right)$ that was calculated using our experimental lattice parameters (Figure 3B) at selected temperatures in the Pnma phase $(T=65,300,424,515$,
$607,703$, and $752 \mathrm{~K})$ and the $C m c m$ phase $(T=799,888$, and $970 \mathrm{~K})$, respectively. In the Pnma phase, the highest VB peak appears in the $\Gamma-Z$ direction (VB2), parallel to the $c$ direction (shown on an expanded scale in Figure 4B). As the temperature decreases from $752 \mathrm{~K}$, this peak splits into two ("pudding mold bands" which lead to a large Seebeck coefficient) ${ }^{30}$ with the left peak moving toward the $E_{\mathrm{F}}$ with 

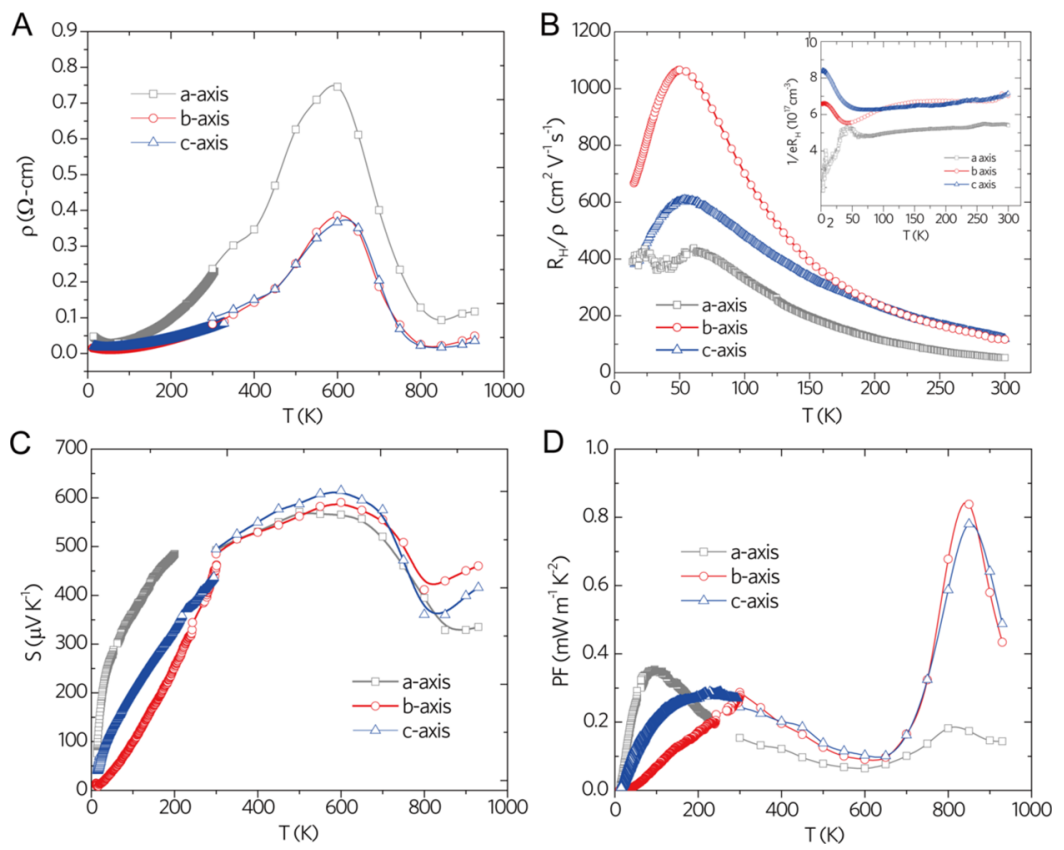

Figure 5. Electronic transport of SnSe. (A) Electrical resistivity $(\rho)$ of SnSe as a function of temperature. (B) Carrier mobility $\left(\mu=R_{\mathrm{H}} / \rho\right)$, and $1 /$ $e R_{\mathrm{H}}$ shown in the inset figure as a function of temperature. (C) Seebeck coefficient $(S)$ and (D) TE power factor $\left(\mathrm{PF}=S^{2} / \rho\right)$ of $\mathrm{SnSe}$ as a function of temperature.

decreasing temperature. Incidentally, the unique pudding mold-shaped VB was reported through high-resolution ARPES measurements that revealed two ellipsoid hole pockets along the $\Gamma-Z$ direction close to $E_{\mathrm{F}}$, in good agreement with the metallic transport of these samples. ${ }^{17}$ The second VB peak (VB1) (shown on an expanded scale in Figure 4B), which is parabolic and along the $\Gamma-Y$ direction, moves toward $E_{\mathrm{F}}$ with increasing temperature. It is worth noting that these two $\mathrm{VB}$ peaks shift in an opposite manner with temperature relative to $E_{\mathrm{F}}$, which influence the carrier concentration of SnSe. In contrast to $\mathrm{VB}$, the conduction band minimum (CBM) in the $\Gamma-Y$ direction $(\mathrm{CB} 1)$ moves away from the $E_{\mathrm{F}}$ with increasing temperature and becomes closer in energy to the broad CBM at $\Gamma$ point (CB2), which causes a band convergence above 600 $\mathrm{K}$ that enhances the $S$ and/or PF of $\mathrm{SnSe}^{3}$ It is expected that these temperature-induced shifts of CBM and VBM will influence the electrical transport properties of SnSe. On the other hand, a direct energy band gap along the $P-Z$ direction is found for $\mathrm{SnSe}$ in the $\mathrm{Cmcm}$ phase (Figure 4C). Generally, the energy band gap of a semiconductor decreases with increasing temperature according to the semiempirical Varshni relation, ${ }^{31}$ we find that the calculated band gap of $\mathrm{SnSe}$ is valley-dependent in both the Pnma and $\mathrm{Cmcm}$ phases and increases monotonically with increasing temperature (Figure 4D).

The temperature-dependent electrical resistivity $(\rho=1 / \sigma)$ of SnSe single crystals along the three major crystallographic directions are shown in Figure 5A. The $\rho$ values are similar in magnitude along the in-plane $b$ and $c$ directions but higher along the perpendicular $a$ direction. Moreover, $\rho$ exhibits an upturn with decreasing temperature below $50 \mathrm{~K}$, which is most pronounced along the $a$ direction that has also been observed by Wang et al. ${ }^{17}$ and attributed to weak localization. In the 50-600 $\mathrm{K}$ temperature range, $\rho$ increases with increasing temperature which can be attributed to the contraction of the VB2 (Figure 4B) that reduces the Fermi surface. The contraction of VB2 also counterbalances the thermal excitation effect that makes the effective carrier concentration become almost temperature-independent for $T>50 \mathrm{~K}$ (inset in Figure 5B). Above $600 \mathrm{~K}, \rho$ decreases by more than an order of magnitude because of the quick upshift of the VB1 with increasing temperature that greatly enlarges the Fermi surface. In other words, $\rho$ of Pnma phase is governed by the temperature-dependent shift and curvature variations of both VB1 and VB2 (Figure 4B).

To gain more insights into the underlying mechanisms, we also measured the Hall coefficient $R_{\mathrm{H}}$ along all three crystallographic directions in the $2-300 \mathrm{~K}$ range (inset of Figure 5B). In an agreement with previous study ${ }^{7}$ and our temperature-dependent band structure calculations (Figure 4), the Arrhenius thermal excitation behavior (which refers to thermal activation of carriers over a band gap) was not observed in the $2-300 \mathrm{~K}$ range. Moreover, the measured Hall coefficients exhibited anisotropy, which is consistent with the anisotropic and multiband nature of the band structure. Between 70 and $300 \mathrm{~K}$, a weak temperature dependence was observed in $1 / e R_{\mathrm{H}}$ along all three directions with a value of 1 / $e R_{\mathrm{H}} \approx 6-7 \times 10^{17} \mathrm{~cm}^{-3}$ along the $b$ and $c$ directions, and a $1 /$ $e R_{\mathrm{H}} \approx 5-6 \times 10^{17} \mathrm{~cm}^{-3}$ along the a direction. The temperature-dependent $1 / e R_{\mathrm{H}}$ below $50 \mathrm{~K}$ exhibits two dissimilar trends with decreasing temperature along the three crystallographic directions. The decrease in $1 / e R_{\mathrm{H}}$ value with decreasing temperature along the $a$ direction is the reason for the slight resistivity upturn along the $a$ direction discussed in Figure 5A. Such complex and anisotropic temperaturedependent behavior along the $a$ direction can be attributed to the weak localization effect. ${ }^{17}$

The Hall mobility $\mu\left(=R_{\mathrm{H}} / \rho\right)$ as a function of temperature between 2 and $300 \mathrm{~K}$ is shown in Figure 5B. In general, $\mu$ exhibits a peak at $50 \mathrm{~K}$ and similar temperature dependence along all three crystallographic directions above $50 \mathrm{~K}$, which is indicative of a dominant electron-phonon coupling. The $\mu$ 
values at $50 \mathrm{~K}(300 \mathrm{~K})$ are $396(52), 1065$ (116), and 602 (118) $\mathrm{cm}^{2} \mathrm{~V}^{-1} \mathrm{~s}^{-1}$ along the $a, b$, and $c$ directions, respectively. Moreover, strong anisotropy is observed in $\mu$ below $50 \mathrm{~K}$, which crossed over to a noisy plateau at $\sim 400 \mathrm{~cm}^{2} \mathrm{~V}^{-1} \mathrm{~s}^{-1}$ along $a$ direction and decreased steeply with a decreasing temperature along the $b$ and $c$ directions. As shown in Figure 5C, the Seebeck coefficient $S$ of $\mathrm{SnSe}$ is positive throughout the entire temperature range, indicative of dominant hole conduction. Along the three crystallographic directions, the $S$ increases with increasing temperature from $\sim 15 \mathrm{~K}$ until the maximum value of $\sim 540-600 \mu \mathrm{V} \mathrm{K}^{-1}$ is reached in the vicinity of $600 \mathrm{~K}$. There is no discernible anomaly near $50 \mathrm{~K}$, in contrast to the observed temperature dependence of $\rho$ (cf. Figure 5A) and $\mu$ (cf. Figure 5B). We employed the Goldsmid-Sharp relation to estimate the energy band gap $E_{\mathrm{g}}$ $=2 e S_{\max } T_{\text {Smax }}{ }^{32}$ where $S_{\max }$ is the peak value of $S$ and $T_{\text {Smax }}$ is the corresponding temperature. The estimated $E_{\mathrm{g}}$ values are found to be in the range $0.62-0.72 \mathrm{eV}$, consistent with the calculated band gap at $600 \mathrm{~K}$ (cf. Figure 4D). The temperature dependence of $S$ is anisotropic, reflecting the anisotropy of the Fermi surface topology of SnSe (Figure 4A-C). Apparently, this anisotropy becomes stronger with decreasing temperature, which is consistent with the observed variations in $1 / e R_{\mathrm{H}}$ (cf. the inset of Figure 5B). Furthermore, $S$ is observed to increase gradually with increasing temperature in the $\mathrm{Cmcm}$ phase, contrary to the expectation that $S$ should decrease above the phase transition temperature. ${ }^{19}$ There are several factors that could affect the thermopower and resistivity simultaneously in a multivalley material system such as $\mathrm{SnSe}$, including effective mass of band valleys, inter-/intra-valley scattering and band gap that needs further investigation.

The temperature-dependent $\mathrm{PF}\left(=S^{2} / \rho\right)$ of our singlecrystalline $\mathrm{SnSe}$ along the three major crystallographic directions from $\sim 15-900 \mathrm{~K}$ is shown in Figure 5D. At $\sim 600$ $\mathrm{K}$, the PF shows a local minimum because of the high $\rho$. At $T$ $\approx 850 \mathrm{~K}$, the higher symmetry $\mathrm{Cmcm}$ phase exhibited a

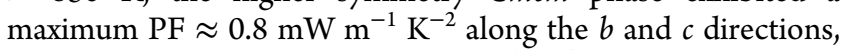

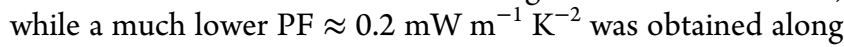
the $a$ direction. These $\mathrm{PF}$ values are comparable to the values $\left(\sim 0.8-1.0 \mathrm{~mW} \mathrm{~m}^{-1} \mathrm{~K}^{-2}\right)$ reported in ref 7 along the $b$ and $c$ directions, but not in agreement with the PF values reported in ref 14.

2.5. Thermal Transport and ZT of Fully Dense SnSe. The total thermal conductivity $\kappa$ of our single-crystalline $\mathrm{SnSe}$ in the temperature range from $25-930 \mathrm{~K}$ is shown in Figure 6A. The solid (open) symbols are values obtained using steady-state (transient laser flash) technique for rod (disc) shaped pieces cut along specific crystallographic directions. Owing to the predominantly high $\rho \approx 0.1-0.8 \Omega \mathrm{cm}$ in the $25-930 \mathrm{~K}$ temperature range (Figure $5 \mathrm{~A}$ ), it is evident that $\kappa$ $\approx \kappa_{\mathrm{L}}$. As shown in the inset of Figure $6 \mathrm{~A}$, well-defined Umklapp peaks are observed at $\sim 30 \mathrm{~K}$ (i.e., $\sim 1 / 7$ of $\Theta_{\mathrm{D}}$ ) along the $b$ and $c$ directions but are absent along the $a$ direction. The $\kappa_{\mathrm{L}}$ of the Pnma phase is inversely proportional to temperature above $30 \mathrm{~K}$, indicating that the Umklapp process is dominant with increasing temperature. The absence of the Umklapp peak along the $a$ direction is presumably due to the downshift of this peak to a lower temperature as the Brillouin zone is substantially shorter along the $a\left(a^{*}\right)$ direction. It is well known that the presence of defects will lower the Umklapp peak intensity and concomitantly broaden it. Ibrahim et al. ${ }^{14}$ and Wang et al. ${ }^{15}$ have reported a stronger Umklapp peak in their studies, implying that the Umklapp peak in this study may
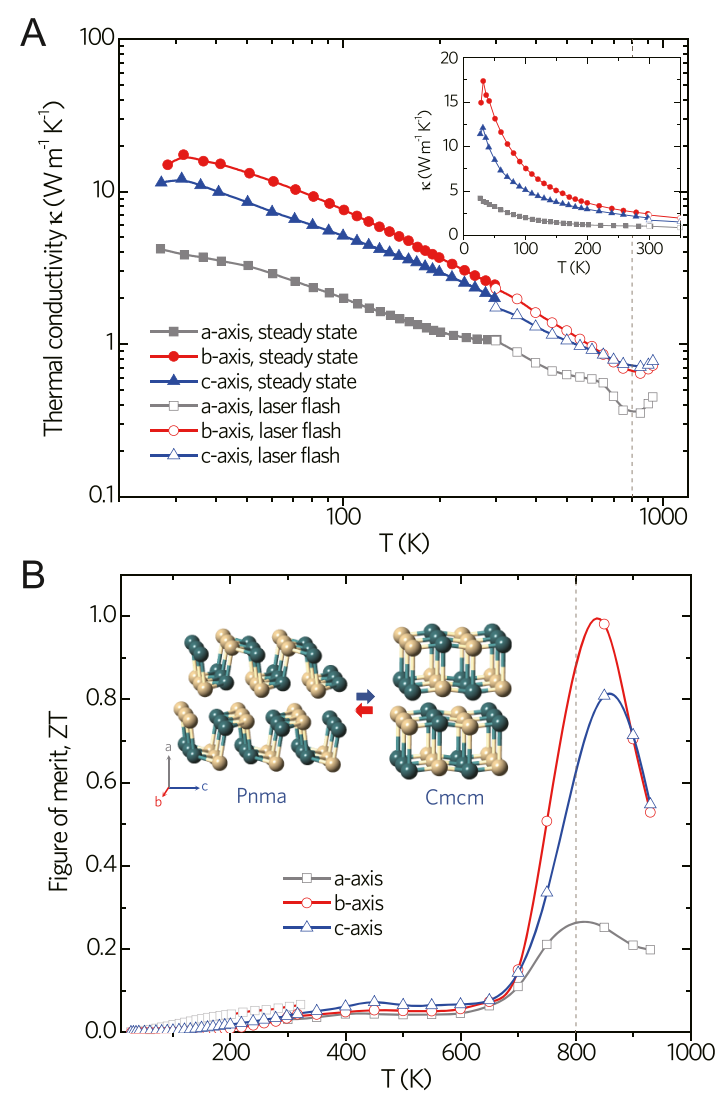

Figure 6. Thermal transport and figure-of-merit of SnSe. (A) Total thermal conductivity $(\kappa)$ plotted on logarithmic and linear (inset figure) scales. The kink at $300 \mathrm{~K}$ along the $a$ direction is a remnant of the radiation losses. (B) TE figure-of-merit of SnSe as a function of temperature. The solid and open symbols in the upper panel correspond to measurements using the steady-state and laser flash techniques, respectively.

arise because of the presence of $<0.35$ at. $\% \operatorname{Sn}(\mathrm{IV})$. Of particular note are the room-temperature values of $\kappa$ along the $b$ and $c$ directions (2.16 and $1.62 \mathrm{~W} \mathrm{~m}^{-1} \mathrm{~K}^{-1}$, respectively), which are comparable to the corresponding values reported by Wasscher et al. ${ }^{33}\left(\kappa \approx 1.9 \mathrm{~W} \mathrm{~m}^{-1} \mathrm{~K}^{-1}\right.$ in the $b c$-plane $)$ and Ibrahim et al. ${ }^{14}$ as well as the theoretical results by Carrete et al. ${ }^{34}$ Compared with the Pnma phase of SnSe, the $\kappa$ of the Cmcm phase is $\sim 0.7-0.9 \mathrm{~W} \mathrm{~m}^{-1} \mathrm{~K}^{-1}$ along $b$ and $c$ directions and $\sim 0.3-0.4 \mathrm{~W} \mathrm{~m}^{-1} \mathrm{~K}^{-1}$ along $a$ direction. The figure-ofmerit, $Z T$ of our single-crystalline $\mathrm{SnSe}$ in the temperature range $25-900 \mathrm{~K}$ is shown in Figure 6B. It is noteworthy that the $Z T$ peaks are present at $850 \mathrm{~K}$ in the $\mathrm{Cmcm}$ phase, which is $\sim 50 \mathrm{~K}$ above $T_{\mathrm{c}}$. Because of the uncertainty of determining $C_{\mathrm{p}}$ value at $T_{\mathcal{O}}$ and subsequently $\kappa$, the plot in Figure $6 \mathrm{~B}$ does not include $Z T$ data points around $T_{\mathrm{c}}$. Despite obtaining similar PF values to that reported in ref 7 , we obtained peak $Z T$ values of $\sim 1.0, \sim 0.8$, and $\sim 0.25$ along the $b, c$, and $a$ directions at $850 \mathrm{~K}$, respectively. Clearly, the $Z T$ values are a factor of two lower than that of $\sim 2.6$ reported in ref 7 , a discrepancy that has been discussed in our previous publication. ${ }^{8}$

\section{CONCLUSION}

Our detailed temperature-dependent structural, electrical, and thermal transport on high-quality single-crystalline $\mathrm{SnSe}$ indicate a low-carrier-concentration multiband feature below $600 \mathrm{~K}$, above which a semiconducting behavior is exhibited. 
The small but robust Fermi surface in SnSe could arise from the presence of $\mathrm{Sn}$ vacancies, which are the dominant intrinsic defects in SnSe. Nonetheless, extensive Mössbauer spectroscopy verify the high quality of our $\mathrm{SnSe}$ crystals and found that the defect concentration is $<0.35$ at. $\% \mathrm{Sn}(\mathrm{IV})$.

\section{EXPERIMENTAL SECTION}

4.1. Synthesis and Characterization. High-purity $\mathrm{Sn}$ chunks (99.999\%) and Se shots (99.999\%) were weighed in the stoichiometric ratio of $\mathrm{SnSe}$, sealed in evacuated silica tubes, and heated at $1223 \mathrm{~K}$ for $48 \mathrm{~h}$ followed by furnacecooling to room temperature. The obtained ingots were crushed and sealed in double silica tubes as it is well known that the volume expansion of the SnSe lattice (along $b$ axis) near the phase transition temperature could break the inner silica tube, causing SnSe to oxidize. The SnSe single crystals were grown using the vertical Bridgman method with a growth rate of $1.5 \mathrm{~mm} \mathrm{~h}^{-1}$ at $1223 \mathrm{~K}$. Finally, high-quality SnSe single crystals (Figure $1 \mathrm{~A}$, weight $\sim 50 \mathrm{~g}$, diam $\approx 13 \mathrm{~mm}$ ) were obtained with a packing density which is $>99 \%$ of the theoretical density, as discussed in the next section. The absence of the $\mathrm{SnO}_{2}$ phase in our SnSe single crystals was confirmed by Raman and Mössbauer spectroscopy. Furthermore, the pole figure and orientation were determined by a PANalytical X'Pert PRO MRD high-resolution X-ray diffractometer. A Rigaku TTRAX 3 X-ray diffractometer $(\lambda=$ $1.54056 \AA$, from $65-300 \mathrm{~K})$ and the synchrotron X-ray beamline BL01C2 $(\lambda=0.619925 \AA$, from $300-1014 \mathrm{~K}$, at the National Synchrotron Radiation Research Center, Taiwan) were employed in conjunction with the General Structure Analysis System (GSAS) program to characterize our SnSe single crystals. ${ }^{35}$ The neutron rocking curve measurements were conducted on BT-7 triple-axis spectrometers at the NIST Center for Neutron Research using pyrolytic graphite PG(002) monochromator crystals to select an incident wavelength of $\lambda$ $=2.359 \mathrm{~A}^{\circ}$, with a PG filter to suppress higher-order wavelength contaminations. Single-detector measurements were made with a PG(002) energy analyzer.

The electrical resistivity $\rho$ and Seebeck coefficient $S$ were measured using the ULVAC ZEM-3 system in the temperature range of $300-900 \mathrm{~K}$. The low temperature $(10-300 \mathrm{~K})$ resistivity and Seebeck coefficient were measured simultaneously using a custom-designed measurement system. ${ }^{36}$ The Hall coefficient $R_{\mathrm{H}}$ and the isobaric heat capacity $C_{\mathrm{p}}$ were measured using a commercial Quantum Design Physical Properties Measurement System in the temperature range of $2-300 \mathrm{~K}$ while a ${ }^{3} \mathrm{He}$ cryostat was used to measure $C_{\mathrm{p}}$ between 0.45 and $2 \mathrm{~K}$. The Hall mobility $\mu$ was calculated using the relation, $\mu=R_{\mathrm{H}} / \rho$ in the context of the Drude model. The total thermal conductivity $\kappa$ in the temperature range of 15$300 \mathrm{~K}$ was measured using a custom-designed steady-state technique. ${ }^{37}$ The thermal conductivity at low temperatures was corrected for radiation losses above $200 \mathrm{~K}$, using the StefanBoltzmann power law. ${ }^{37}$ Above room temperature, the total thermal conductivity was estimated as $\kappa=D_{\mathrm{T}} C_{\mathrm{v}} d$, where the thermal diffusivity $D_{\mathrm{T}}$ was measured by the laser flash method using Netzsch LFA457, $C_{\mathrm{p}}$ was measured using Netzsch Pegasus 404 high-temperature differential scanning calorimetry system, and the packing density $d$ of the crystal was measured at room temperature by the Archimedes method and gas pycnometry (Micromeritics AccuPyc 1330). For a strongly anharmonic material such as $\mathrm{SnSe}, C_{\mathrm{p}}>C_{\mathrm{v}}$ at elevated temperatures but the discrepancy between $C_{p}$ and $C_{v}$ is offset to the first order by a decrease in the packing density because of thermal expansion. Hence, $C_{\mathrm{p}} \approx C_{\mathrm{v}}$ except at $T_{\mathrm{c}}$. As described above, the low- and high-temperature thermal conductivity values are measured using different principles, sample geometries, and measurement environments. These techniques offer an opportunity to probe the presence of cracks in the SnSe single crystals. During the laser flash measurement, the cracks may leak the laser signal and yield an artificially high thermal diffusivity value, however, if the same cracks were present under a dynamic vacuum, no heat could conduct through the cracks during the low temperature steady-state measurements. Thus one would expect to see a mismatch between the results of low- and high-temperature measurements in the presence of cracks. As shown in Figure 6A, the low- and high-temperature measurements match exceedingly well; thus, the presence of cracks can be excluded in our SnSe single crystals. The surface topography of in situ cleaved singlecrystalline SnSe was collected using an etched tungsten wire in a custom-designed ${ }^{3} \mathrm{He}$ STM. ${ }^{38}$

Room-temperature ${ }^{119} \mathrm{Sn}$ Mössbauer spectra were obtained in transmission geometry using a MS-96 constant acceleration drive (Palacky University, Olomouc) with a $10 \mathrm{mCi}$ $\mathrm{Ca}^{119 \mathrm{~m}} \mathrm{SnO}_{3}$ source. The spectrometer was calibrated using $\alpha$-iron foil with a $1 \mathrm{mCi}{ }^{57} \mathrm{Co}$ source in rhodium matrix. The SnSe sample was prepared by mechanically exfoliating the crystals on aluminized Mylar tape, wherein two layers were used for the SnSe samples. The radiation was parallel to the crystallographic $a$ axis. The isomer shift is reported relative to $\mathrm{SnO}_{2}$ at ambient temperature. The total collected statistics for the sample was $24 \times 10^{6}$ counts per channel, after binning to a total of 256 channels, collected for 5 days, for SnSe. The signalto-noise ratio was about the same for both samples as the latter samples had twice the thickness and absorption. An appropriate statistics estimator for a fit of one data set by two models with different number of parameters is given by the Akaike Information Criterion, ${ }^{39,40}$ which yields relative likelihood for the 1 component model of $\exp \left(\left(\mathrm{AIC}_{1}-\mathrm{AIC}_{2}\right) /\right.$ $2)<0.001$, where $\mathrm{AIC}=2 \times k+N \ln (\mathrm{SSR} / \mathrm{N})$.

\section{ASSOCIATED CONTENT}

\section{Supporting Information}

The Supporting Information is available free of charge on the ACS Publications website at DOI: 10.1021/acsomega.8b03323.

$$
\text { First-principles DFT calculations }{ }^{41-49} \text { (PDF) }
$$

\section{AUTHOR INFORMATION}

\section{Corresponding Authors}

*E-mail: cheny2@phys.sinica.edu.tw (Y.-Y.C.).

*E-mail: arao@clemson.edu (A.M.R.).

ORCID $\odot$

Wen-Hsien Li: 0000-0003-1594-9879

Raphael P. Hermann: 0000-0002-6138-5624

Apparao M. Rao: 0000-0002-1450-3499

\section{Funding}

Authors Y.-F.L. and J.H. acknowledge the financial support of NSF DMR 1307740. Mössbauer spectroscopy by R.P.H. was supported by the U.S. Department of Energy, Office of Science, Basic Energy Sciences, Materials Sciences and Engineering Division. Y.-Y.C. would like to acknowledge the financial support of Academia Sinica no. AS-SS-106-01-1. 


\section{Notes}

The authors declare no competing financial interest.

\section{ACKNOWLEDGMENTS}

The authors wish to acknowledge Prof. Terry Tritt (Clemson University) for providing access to low temperature measurement facilities in his laboratory, and Prof. Julian Goldsmid, Dr. Pooja Puneet, and Prakash Parajuli for valuable discussions. We also thank National Institute of Standards and Technology and Dr. Jeffrey W. Lynn for providing the neutron beam time that made the neutron rocking curve measurements possible. R.P.H. thanks Dipanshu Bansal and Jiawang Hong for numerical phonon data in refs 11 and 26.

\section{REFERENCES}

(1) Hicks, L. D.; Dresselhaus, M. S. Effect of Quantum-Well Structures on the Thermoelectric Figure of Merit. Phys. Rev. B: Condens. Matter Mater. Phys. 1993, 47, 12727-12731.

(2) Snyder, G. J.; Toberer, E. S. Complex Thermoelectric Materials. Nat. Mater. 2008, 7, 105-114.

(3) Pei, Y.; Shi, X.; Lalonde, A.; Wang, H.; Chen, L.; Snyder, G. J. Convergence of Electronic Bands for High Performance Bulk Thermoelectrics. Nature 2011, 473, 66-69.

(4) Liu, Y.; Zhou, M.; He, J. Towards Higher Thermoelectric Performance of $\mathrm{Bi}_{2} \mathrm{Te}_{3}$ via Defect Engineering. Scr. Mater. 2016, 111, 39-43.

(5) Morelli, D. T.; Jovovic, V.; Heremans, J. P. Intrinsically Minimal Thermal Conductivity in Cubic I-V-VI ${ }_{2}$ Semiconductors. Phys. Rev. Lett. 2008, 101, 035901.

(6) Cohn, J. L.; Nolas, G. S.; Fessatidis, V.; Metcalf, T. H.; Slack, G. A. Glasslike Heat Conduction in High-Mobility Crystalline Semiconductors. Phys. Rev. Lett. 1999, 82, 779-782.

(7) Zhao, L.-D.; Lo, S.-H.; Zhang, Y.; Sun, H.; Tan, G.; Uher, C.; Wolverton, C.; Dravid, V. P.; Kanatzidis, M. G. Ultralow Thermal Conductivity and High Thermoelectric Figure of Merit in SnSe Crystals. Nature 2014, 508, 373-377.

(8) Wei, P.-C.; Bhattacharya, S.; He, J.; Neeleshwar, S.; Podila, R.; Chen, Y. Y.; Rao, A. M. The Intrinsic Thermal Conductivity of SnSe. Nature 2016, 539, E1-E2.

(9) Chattopadhyay, T.; Pannetier, J.; Von Schnering, H. G. Neutron Diffraction Study of the Structural Phase Transition in SnS and SnSe. J. Phys. Chem. Solids 1986, 47, 879-885.

(10) Li, C. W.; Hong, J.; May, A. F.; Bansal, D.; Chi, S.; Hong, T.; Ehlers, G.; Delaire, O. Orbitally Driven Giant Phonon Anharmonicity in SnSe. Nat. Phys. 2015, 11, 1063-1069.

(11) Bansal, D.; Hong, J.; Li, C. W.; May, A. F.; Porter, W.; Hu, M. Y.; Abernathy, D. L.; Delaire, O. Phonon Anharmonicity and Negative Thermal Expansion in SnSe. Phys. Rev. B: Condens. Matter Mater. Phys. 2016, 94, 054307.

(12) Sassi, S.; Candolfi, C.; Vaney, J.-B.; Ohorodniichuk, V.; Masschelein, P.; Dauscher, A.; Lenoir, B. Assessment of the Thermoelectric Performance of Polycrystalline p-Type SnSe. Appl. Phys. Lett. 2014, 104, 212105.

(13) Chen, C.-L.; Wang, H.; Chen, Y.-Y.; Day, T.; Snyder, G. J. Thermoelectric Properties of p-Type Polycrystalline SnSe Doped with Ag. J. Mater. Chem. A 2014, 2, 11171-11176.

(14) Ibrahim, D.; Vaney, J.-B.; Sassi, S.; Candolfi, C.; Ohorodniichuk, V.; Levinsky, P.; Semprimoschnig, C.; Dauscher, A.; Lenoir, B. Reinvestigation of the Thermal Properties of SingleCrystalline SnSe. Appl. Phys. Lett. 2017, 110, 032103.

(15) Wang, S.; Hui, S.; Peng, K.; Bailey, T. P.; Liu, W.; Yonggao, Y.; Xiaoyuan, Z.; Tang, X.; Uher, C. Low Temperature Thermoelectric Properties of p-Type Doped Single-Crystalline SnSe. Appl. Phys. Lett. 2018, 112, 142102 .

(16) Nguyen, V. Q.; Kim, J.; Cho, S. A Review of SnSe: Growth and Thermoelectric Properties. J. Korean Phys. Soc. 2018, 72, 841-857.
(17) Wang, Z.; Fan, C.; Shen, Z.; Hua, C.; Hu, Q.; Sheng, F.; Lu, Y.; Fang, H.; Qiu, Z.; Lu, J.; et al. Defects Controlled Hole Doping and Multivalley Transport in SnSe Single Crystals. Nat. Commun. 2018, 9, $1-9$

(18) Shi, G.; Kioupakis, E. Quasiparticle Band Structures and Thermoelectric Transport Properties of p-Type SnSe. J. Appl. Phys. 2015, 117, 065103

(19) Kutorasinski, K.; Wiendlocha, B.; Kaprzyk, S.; Tobola, J. Electronic Structure and Thermoelectric Properties of $\mathrm{n}$ - and $\mathrm{p}$-Type SnSe from First Principles Calculations. Phys. Rev. B: Condens. Matter Mater. Phys. 2015, 91, 205201.

(20) Guo, R.; Wang, X.; Kuang, Y.; Huang, B. First-Principles Study of Anisotropic Thermoelectric Transport Properties of IV-VI Semiconductor Compounds SnSe and SnS. Phys. Rev. B: Condens. Matter Mater. Phys. 2015, 92, 115202.

(21) Duvjir, G.; Min, T.; Thi Ly, T.; Kim, T.; Duong, A.-T.; Cho, S.; Rhim, S. H.; Lee, J.; Kim, J. Origin of p-Type Characteristics in a SnSe Single Crystal. Appl. Phys. Lett. 2017, 110, 262106.

(22) Taniguchi, M.; Johnson, R. L.; Ghijsen, J.; Cardona, M. Core Excitons and Conduction-Band Structures in Orthorhombic GeS, GeSe, SnS, and SnSe Single Crystals. Phys. Rev. B: Condens. Matter Mater. Phys. 1990, 42, 3634-3643.

(23) Baggio, E. M.; Sonnino, T. Isomer Shift and Electronegativity in Compounds of Tin and Group-VI Elements. J. Chem. Phys. 1970, 52, 3786.

(24) Karyagin, S. V. The Possible Reason for the Asymmetry of Doublet Components of Mossbauer's Absorption Spectrum in Certain Powdery Tin Compounds. Dokl. Akad. Nauk SSSR 1963, $148,1102-1105$.

(25) Lippens, P. E. Interpretation of the ${ }^{119} \mathrm{Sn}$ Mössbauer isomer shifts in complex tin chalcogenides. Phys. Rev. B: Condens. Matter Mater. Phys. 1999, 60, 4576-4586.

(26) Ding, Y.; Xiao, B.; Tang, G.; Hong, J. Transport Properties and High Thermopower of $\mathrm{SnSe}_{2}$ : A Full Ab-Initio Investigation. J. Phys. Chem. C 2017, 121, 225-236.

(27) Buckingham, M. J.; Fairbank, W. M. Chapter III The Nature of the $\lambda$-Transition in Liquid Helium. Prog. Low Temp. Phys. 1961, 3, $80-112$.

(28) Phillips, N. E. Low-Temperature Heat Capacity of Metals. CRC Crit. Rev. Solid State Sci. 1971, 2, 467-553.

(29) Dewandre, A.; Hellman, O.; Bhattacharya, S.; Romero, A. H.; Madsen, G. K. H.; Verstraete, M. J. Two-Step Phase Transition in SnSe and the Origins of Its High Power Factor from First Principles. Phys. Rev. Lett. 2016, 117, 276601.

(30) Kuroki, K.; Arita, R. "Pudding Mold" Band Drives Large Thermopower in $\mathrm{Na} \mathrm{XCoO}_{2}$. J. Phys. Soc. Jpn. 2007, 76, 083707.

(31) Varshni, Y. P. Temperature Dependence of the Energy Gap in Semiconductors. Physica 1967, 34, 149-154.

(32) Goldsmid, H. J.; Sharp, J. W. Estimation of the Thermal Band Gap of a Semiconductor from Seebeck Measurements. J. Electron. Mater. 1999, 28, 869-872.

(33) Wasscher, J. D.; Albers, W.; Haas, C. Simple Evaluation of the Maximum Thermoelectric Figure of Merit, with Application to Mixed Crystals $\mathrm{SnS}_{(1-\mathrm{x})} \mathrm{Se}_{\mathrm{x}}$. Solid-State Electron. 1963, 6, 261-264.

(34) Carrete, J.; Mingo, N.; Curtarolo, S. Low Thermal Conductivity and Triaxial Phononic Anisotropy of SnSe. Appl. Phys. Lett. 2014, 105, 101907.

(35) Von Dreele, R.; Larson, A. General Structure Analysis System (GSAS), Los Alamos National Laboratory Report LAUR, 2004; Vol. 748, pp 86-748.

(36) Pope, A. L.; Littleton, R. T.; Tritt, T. M. Apparatus for the rapid measurement of electrical transport properties for both "needlelike” and bulk materials. Rev. Sci. Instrum. 2001, 72, 3129.

(37) Pope, A. L.; Zawilski, B.; Tritt, T. M. Description of Removable Sample Mount Apparatus for Rapid Thermal Conductivity Measurements. Cryogenics 2001, 41, 725-731.

(38) Guan, S.-Y.; Chen, P.-J.; Chu, M.-W.; Sankar, R.; Chou, F.; Jeng, H.-T.; Chang, C.-S.; Chuang, T.-M. Superconducting Topo- 
logical Surface States in the Noncentrosymmetric Bulk Superconductor $\mathrm{PbTaSe}_{2}$. Sci. Adv. 2016, 2, el600894.

(39) Akaike, H. A new look at the statistical model identification.

IEEE Trans. Autom. Control 1974, 19, 716-723.

(40) Burnham, K. P.; Anderson, D. R. Multimodel Inference. Sociol. Methods and Res. 2004, 33, 261-304.

(41) Hohenberg, P.; Kohn, W. Inhomogeneous Electron Gas. Phys. Rev. 1964, 136, B864-B871.

(42) Kresse, G.; Hafner, J. Ab initiomolecular-dynamics simulation of the liquid-metal-amorphous-semiconductor transition in germanium. Phys. Rev. B: Condens. Matter Mater. Phys. 1994, 49, 1425114269.

(43) Kresse, G.; Furthmüller, J. Efficiency of Ab-Initio Total Energy Calculations for Metals and Semiconductors Using a Plane-Wave Basis Set. Comput. Mater. Sci. 1996, 6, 15-50.

(44) Blöchl, P. E. Projector Augmented-Wave Method. Phys. Rev. B: Condens. Matter Mater. Phys. 1994, 50, 17953-17979.

(45) Kresse, G.; Joubert, D. From ultrasoft pseudopotentials to the projector augmented-wave method. Phys. Rev. B: Condens. Matter Mater. Phys. 1999, 59, 1758.

(46) Perdew, J. P.; Burke, K.; Ernzerhof, M. Generalized Gradient Approximation Made Simple. Phys. Rev. Lett. 1996, 77, 3865-3868.

(47) Shishkin, M.; Kresse, G. Self-Consistent GW Calculations for Semiconductors and Insulators. Phys. Rev. B: Condens. Matter Mater. Phys. 2007, 75, 235102.

(48) Godby, R. W.; Schlüter, M.; Sham, L. J. Self-Energy Operators and Exchange-Correlation Potentials in Semiconductors. Phys. Rev. B: Condens. Matter Mater. Phys. 1988, 37, 10159-10175.

(49) Shishkin, M.; Kresse, G. Implementation and Performance of the Frequency-Dependent GW Method within the PAW Framework. Phys. Rev. B: Condens. Matter Mater. Phys. 2006, 74, 35101. 